\title{
$\mathrm{Au}$ détour du moment Guizot
}

Les « Voyages en France » ou le Tableau de la France de Stendhal At the Detour of the Guizot's Moment: "Travels Through France" or Stendhal's Tableau de la France

\section{Laure Lévêque}

\section{OpenEdition}

\section{Journals}

\section{Édition électronique}

URL : http://journals.openedition.org/recherchestravaux/895

ISSN : 1969-6434

Éditeur

UGA Éditions/Université Grenoble Alpes

Édition imprimée

ISBN : 978-2-37747-006-8

ISSN : 0151-1874

Référence électronique

Laure Lévêque, "Au détour du moment Guizot », Recherches \& Travaux [En ligne], 90 | 2017, mis en ligne le 15 juin 2017, consulté le 08 septembre 2020. URL : http://journals.openedition.org/ recherchestravaux/895

Ce document a été généré automatiquement le 8 septembre 2020

(C) Recherches \& Travaux 


\title{
Au détour du moment Guizot
}

\author{
Les « Voyages en France » ou le Tableau de la France de Stendhal \\ At the Detour of the Guizot's Moment: "Travels Through France" or Stendhal's \\ Tableau de la France
}

Laure Lévêque

1 Le postulat sur lequel reposera mon propos est que l'important ensemble que forment les «Voyages en France » de Stendhal - les Mémoires d'un touriste, seul de ces textes à avoir été publié du vivant de Stendhal, en 1838, le Voyage en France et le Voyage dans le Midi de la France ${ }^{1}$, de rédaction contemporaine - est inséparable d'un contexte très particulier qui est celui de ce qu'il est convenu d'appeler le "moment Guizot", autrement dit que l'énonciation de ces textes n'aurait pas été concevable avant ces années 1830 où s'ouvre un nouveau rapport à la France et à son histoire, à tout ce qui relève de l'identité nationale qui relance le processus d'inventaire, de réexamen et, finalement, de réappropriation, culturelle et identitaire, ouvert sous la Révolution autour de l'abbé Grégoire et de l'action d'Alexandre Lenoir.

2 Dans ce long processus d'assimilation référentielle, les années 1830, marquées par la recherche d'un consensus national, jouent un rôle moteur et accouchent d'un nouveau rapport entre passé et présent que cristallise le moment Guizot. Parvenu au pouvoir en août 1830, Guizot « fait de l'héritage culturel un enjeu de gouvernement ${ }^{2}$ », le support privilégié d'une cohésion sociale garantie par une fierté nationale puisée à la continuité des gloires et illustrations françaises, qu'il s'agit de réveiller. La création, en octobre 1830, de l'inspection générale des Monuments historiques ${ }^{3}$ sera l'un des instruments de cette politique que Guizot poursuivra, de 1832 à 1837, comme ministre de l'Instruction publique, diligentant des recherches visant à exhumer et à publier des archives inédites «de la littérature, de la philosophie, des sciences et des arts considérés dans leurs rapports avec l'histoire générale de la France » qui mobilisent les meilleurs esprits : Augustin Thierry, Quinet, Michelet ou Sue. Couronnement de l'édifice, Guizot est, avec Thierry et Michelet, du cartel d'historiens qui conseille Louis-Philippe dans son programme de muséalisation du palais de Versailles, entreprise de réconciliation arrêtée dès 1832 et inaugurée en 1837, où éclate, autour de la mémoire collective, 
l'enjeu de l'héritage, pleinement assumé dans un Versailles nationalisé devenu Musée historique dédié « À toutes les gloires de la France ».

3 La publication des Voyages en France intervient donc à un moment charnière de l'historiographie française, qui suit de près cette année 1837 si importante pour la pacification de l'héritage national, qu'il s'agit d'organiser en histoire, ce à quoi concourt la Commission des Monuments historiques, précisément instituée en 1837, et encadrée par des Instructions introduites par une Lettre de Guizot qui désigne l'archéologie comme science auxiliaire de l'histoire, se proposant de dresser une carte monumentale de la France.

\section{Le voyage aux sources de la nation}

Si les premiers voyages de Mérimée, Notes d'un Voyage dans le Midi de la France (1835) et Notes d'un voyage dans l'Ouest de la France (1836), publiés avant ses propres relations, sont abondamment mis à profit par Stendhal, c'est plus largement que les Voyages en France de Stendhal témoignent de ce contexte qui entreprend de redéfinir l'histoire, ses vecteurs et ses marqueurs. Aussi faut-il donner tout son sens au programme de visites que le touriste remplit avec conscience, entre musées (Rennes, Vannes, Lyon, Grenoble, Marseille...) et monuments, et jusqu'aux formes de la sociabilité qui le mettent en contact, via érudits locaux et sociétés savantes, avec le mouvement antiquaire qui participe de ces réaménagements, l'État soutenant les sociétés savantes, dont la plus connue est sans doute la Société française d'archéologie, fondée en 1834 sous l'impulsion d'Arcisse de Caumont.

5 Sa volonté de synthèse, le régime de Juillet ne l'expose nulle part avec plus d'évidence que dans le nouveau musée de L'Histoire de France, autour de chronotopes signifiants que retrouve Michelet dans son Tableau de la France paru en décembre 1833, à l'entame du second volume de L'Histoire de France ${ }^{4}$, qui enregistre et emblématise un renversement de perspective qui renouvelle, sinon redistribue, les termes du récit historique, nourri par l'observation directe des terroirs, de leurs habitants et des mœurs de ceux-ci. Dès 1830, en effet, nouveau responsable de la section historique des Archives, il découvre, outre les archives de province qu'il s'en va recenser, les différents pays de France - Bretagne, Normandie et Ardennes en 1831, Sud-Ouest, Midi et Centre en 1835 - qui font du territoire national le registre vivant d'une histoire accomplie, en cours et à venir. Pour lui, le territoire est alors vécu comme une géographie où le temps s'est territorialisé en histoire, une histoire qui reste à déchiffrer, à lire, à interpréter, avant de pouvoir s'écrire. Désormais, à côté de la tradition érudite fondée sur l'exégèse des textes, s'impose le décryptage du palimpseste inscrit dans le sol, quand émerge la conception moderne, globalisante, d'une histoire où tout fait signe, où toutes les traces doivent trouver leur place ${ }^{5}$. En cela, Michelet opère une transformation fondatrice dont il crédite encore L'Histoire du XIXe siècle en 1874, qui pose : «Je suis né au milieu de la grande révolution territoriale ${ }^{6}$.»

Pour Paule Petitier qui cite Michelet ( «J'ai commencé à être c'est-à-dire à écrire à la fin de 1831 »), "Le Tableau coïncide avec une seconde naissance du $\operatorname{moi}^{7}$ », si bien que, né, il l'est même deux fois, en 1798 et en 1831, deux moments où cerner cet objet mouvant qu'est désormais la France relève d'une épistémologie neuve et appelle des méthodologies renouvelées. 
7 Parallèlement à la mise sur pied d'un étagement de services relevant de l'État chargés d'établir une nomenclature, l'idée de revue qu'implique désormais la conservation, et d'abord l'identification, du patrimoine qui repose dorénavant sur des listes, est assumée par le principe du voyage, qui gouvernait déjà l'entreprise de recensement menée par Millin. Il faut alors procéder à l'inventaire après-révolutionnement du pays. Très vite, des voyages et des enquêtes que l'administration encourage sont entrepris. André Monglond ${ }^{8}$ dénombre ainsi, entre 1789 et 1812, quelque 700 ouvrages consacrés à la re-connaissance de la France dans la diversité de ses pays. Une mosaïque que devait considérablement populariser la série, inaugurée en 1820, des Voyages pittoresques et romantiques dans l'ancienne France, dont le succès colossal allait jeter sur les routes écrivains et amateurs, partie prenante d'une histoire nationale dont chacun entend s'emparer, générant un débat fécond qui engage, par le biais du patrimoine, la notion même d'idée nationale, que la Révolution vient de violemment secouer, imposant d'en reformuler le contenu en récit national.

8 C'est dire que l'examen de la question ne relève pas du supplément d'âme réservé à une petite communauté élitiste d'érudits - auxquels le touriste stendhalien se frotte d'ailleurs volontiers, comme déjà, hors toute fiction, Michelet avant lui dans ses tournées d'inspection, aucun des deux ne négligeant ces sources précieuses dans leur enquête - mais bien d'une urgence véritablement nationale, grosse de l'image de la France, urgence que manifeste aussi l'inflation de tout ce qui concourt à dresser une topographie de la France révolutionnée : gravures qui illustrent les Voyages pittoresques ou statistiques qui accompagnent les Forces productives et commerciales de la France, grand classique de Charles Dupin qui, en 1827 concourt à dresser un premier Tableau de la France avant celui de Michelet, véritable symbiose entre géographie et histoire qui acclimate l'idée d'une relation charnelle entre un territoire et une culture. C'est aussi là que s'origine, au-delà de la démocratisation des transports qui rend la circulation plus aisée - ce dont témoigne notre touriste qui emprunte, de Lyon à Saint-Étienne, la première ligne française de chemin de fer ouverte en 1831 - cette fièvre voyageuse qui saisit la librairie française et s'accompagne toujours d'un regard taxinomique. Tout en suivant la mode, venue du $\mathrm{XVIII}^{\mathrm{e}}$ siècle, des récits viatiques où l'on se promène en terre(s) reçue(s) comme incontestable(s) de culture et de patrimoine, fleurit une littérature d'un type nouveau, mais dont la tonalité ironique ne doit pas masquer le sérieux, dont la série à succès d'Étienne de Jouy réalise le parangon, en promenant ses Hermites jusqu'en province, enfin légitimée, dans une nation désormais une et indivisible, comme une composante du territoire, qui doit comme telle être parcourue. Une double tradition dont ces textes de Stendhal, qui font la part belle à la province, nous paraissent également participer.

\section{L'unité de la patrie au miroir des pays de France}

9 Inséparable de l'invention des sciences historiques qui se joue alors, dans les années 1820-1830, autour des frères Thierry, de Mignet, Quinet, Michelet, Barante, Thiers ou Guizot, qui visent à doter la France, après le cataclysme révolutionnaire et les réfections identitaires qu'il impose, d'une ontologie qui ravaude les déchirures de l'histoire et assure la permanence de soi à soi, c'est bien de cette dynamique que relève ce massif. En témoigne la part que Stendhal réserve à une question qui cristallise l'identité nationale en construction, celle des races à l'origine de la nation France. 
Pleinement relancée à la veille de la Révolution par l'abbé Sieyès qui oppose des Gaulois rattachés à la souche indigène et populaire de la nation à des Francs qui auraient importé la féodalité de Germanie, elle apparaît à nouveau chez Stendhal, dans l'opposition des Kymris, des Gaëls et des Ibères ${ }^{9}$, d'abord défendue par Amédée Thierry dans son Histoire des Gaulois (1828) avant de trouver des échos auprès de William Edwards, dont le frère Edward, un familier de Stendhal, devait fonder, en 1839, la Société d'ethnologie dont Michelet allait être l'un des premiers adhérents. Ce dernier donnait, en 1833, pour assise à son Histoire de France qui fait le départ entre une "Gaule gallique » et une "Gaule kymrique », cette théorie des races venue d'Amédée Thierry ${ }^{10}$ que revitalise Stendhal, moins pour elle-même que pour ce qu'elle ouvre la question des origines, articulant celles de la France à celles de la nation. Ce pourquoi le touriste peut oser ce raccourci saisissant, qui met la question de la Révolution au cœur des débats où elle figure comme préalable à toute analyse :

Je vais aborder la partie la plus difficile de l'étude des trois races d'hommes qui couvrent le sol de la France. Je répète que c'est là le seul remède que je connaisse à cette fatale maladie de la haine impuissante qui nous travaille depuis que le meurtre du maréchal Brune nous a relancés dans la période de sang des révolutions. ( $M T$, p. 299)

10 Au-delà de la validité scientifique de ces théories, l'intérêt de la référence réside dans le rappel que la longue histoire de la France est celle de l'émergence d'une unité. Pour le Michelet de l'Introduction à l'Histoire universelle (1831) déjà, «cette fusion intime des races constitue l'identité de notre nation, sa personnalité » et il s'agit de savoir «quel est le génie propre de cette personnalité multiple » (IHU, p. 51) qui se dessine, fruit d'un syncrétisme que la Révolution n'a fait que mener à son terme, en parachevant la politique d'assimilation patiemment conduite au fil des siècles par le pouvoir royal.

11 Aussi va-t-il s'agir de parcourir les pays de France en y quêtant une marche à l'intégration qui signe la fin des particularismes. Chez Stendhal, le touriste décrète en Arles que «dans cinquante ans peut-être il n'y aura plus de Provençaux ni de langue provençale " (VF, p. 549). En 1832, à Montpellier, on ne " parlait encore que patois, en 1837, on parle français » (VF, p. 553), et «si jamais l'on établit le seul chemin de fer raisonnable, celui de Paris à Marseille, en dix ans le patois provençal et le patois languedocien cessent d'exister " (VF, p. 554). Peu avant, Michelet, à la recherche de facteurs d'unité susceptibles de rendre compte du genius loci, ouvrait son traité sur l'unité linguistique: "L'histoire de France commence avec la langue française. La langue est le signe principal d'une nationalité. » (TF, p. 1.) Qu'il y ait lieu de se féliciter tout uniment de l'alignement et de l'uniformité linguistiques ou non, reste que le propre de la langue est de fonctionner comme un système articulé. Or, c'est bien de cela qu'il va être question. Critère à l'évidence éminemment discriminant pour Stendhal. Il $\mathrm{y}$ a ainsi des usages de la langue ampoulés. À Autun, où il trouve un volume de Balzac dans sa chambre, le touriste le brocarde de bon cœur : «Je voudrais un style plus simple [...]. Je suppose qu'il fait ses romans en deux temps, d'abord raisonnablement, puis il les habille en beau style néologique, avec les patiments de l'âme, il neige dans mon cœur, et autres belles choses.» (MT, p. 34-35.) Lourdeur qui se retrouve dans le paysage disqualifié de la prétendue "belle Touraine», pays de bonne culture, vrai potager, plutôt que "jardin de la France", bien propre à donner "le plus fécond de nos romanciers ». En revanche, cette articulation ménage aussi des pays où le style est nature ${ }^{11}$ si bien que, dans cette nouvelle cartographie de la France, des territoires se 
dessinent progressivement, quand bien même c'est plutôt aux marges de l'espace français, en Lombardie ou du côté d'Irun ${ }^{12}$, qu'il faut en chercher l'illustration.

Sur ce point, Stendhal - faute d'être exactement sur la même ligne que Michelet quant à l'interprétation qu'il convient de retenir de la Révolution et de la traduction de son œuvre émancipatrice dans la vie du pays - demeure donc en deçà des conclusions auxquelles aboutissait Michelet dès 1831, détaillant dans l'Introduction à l'histoire universelle la progressive émergence d'une « France française » :

La puissance de l'assimilation se trouve ici au plus haut degré : la France française a su attirer, absorber, identifier les Frances anglaise, allemande, espagnole, dont elle était environnée. Elle les a neutralisées l'une par l'autre et converties toutes à sa substance. Elle a amorti la Bretagne par la Normandie, la Franche-Comté par la Bourgogne; par le Languedoc, la Guyenne et la Gascogne; par le Dauphiné, la Provence. Elle a méridionalisé le Nord, septentrionalisé le Midi ; a porté au second le génie chevaleresque de la Normandie, de la Lorraine; au premier la forme romaine de la municipalité toulousaine, et l'industrialisme grec de Marseille ${ }^{13}$.

Cette France française, son acte de naissance peut donc être établi en 843, lorsque, avec le traité de Verdun, Charles II le Chauve, s'exprimant en français face à son frère Lothaire, concourt à faire sortir de sa gangue un territoire, la France qui «pour la première fois [...] se produit dans sa forme géographique » : « le pays apparaît, dans ses diversités locales, dessiné par ses montagnes, par ses rivières. Les divisions politiques répondent ici aux divisions physiques » si bien que : «Bien loin qu'il y ait, comme on l'a dit, confusion et chaos, c'est un ordre, une régularité inévitable et fatale.» Fatale, entendons qui tient $\mathrm{du}$ destin quand «Chose bizarre! Nos quatre-vingt-six départements répondent, à peu de chose près, aux quatre-vingt-six capitulaires d'où sont sorties la plupart des souverainetés féodales, et la Révolution, qui venait de donner le dernier coup à la féodalité, l'a imitée malgré elle. Le vrai point de départ de notre histoire doit être une division politique de la France, formée d'après sa division physique et naturelle. L'histoire est d'abord toute géographie » (TF, p. 1-2).

Si les grands traits géomorphologiques qui découpent le paysage sont donnés comme structurants - de même que Michelet invite son lecteur à « contempl[er] l'ensemble de la France pour la voir se diviser elle-même ", convoquant massifs montagneux, bassins fluviaux et « écoulement des races » (TF, p. 3), au début de son périple, qui le mène de Langres à Dijon, Philippe $\mathrm{L}^{* * *}$ administre au sien une longue description des cinq chaînes montagneuses qu'il voit découper la France au motif que seule l'écriture de ces pages lui a fait comprendre le sol français $(M T, p .60)$-, ils recoupent des caractères qui sont aussi esthétiques et moraux. La mollesse géomorphologique rend aussi compte des caractères des peuples et un plat pays portera toujours des habitants qui manquent d'élévation. Stendhal qui, on le sait, n'hésite pas à déplacer les montagnes ${ }^{14}$, ne peut que tomber d'accord avec Michelet pour flétrir les «plaines décolorées du Berry et de la Champagne» (TF, p. 45), «pays, plat, pâle, d'un prosaïsme désolant » où des équivalences toutes stendhaliennes se déploient quand « Troyes est presque aussi laide qu'industrieuse » (TF, p. 60). Alors la géographie physique s'efface sous ce qu'il faut bien appeler l'histoire, ce que Michelet avait posé dès 1831: dans l'histoire de l'évolution, « on voit [...] diminuer la puissance fatale de la nature, et l'influence de race et de climat devenir moins tyrannique » (IHU, p. 7).

15 Alors, la formule fameuse : « Tel le nid, tel l'oiseau, telle la patrie, tel l'homme. Sans une base géographique, les hommes semblent comme marcher en l'air, comme dans les tableaux chinois ", ne relève en rien d'un fixisme mais définit l'une des deux 
asymptotes entre lesquelles s'inscrira la pensée de Michelet, comme celle de Stendhal; l'autre étant exprimée de façon exemplaire dans un versant plus tardif de la pensée micheletienne, qui proclame que «L'humanité s'est faite elle-même par sa propre action. C'est l'homme qui forge sa fortune [...]. Il est son propre Prométhée. Cela tout à coup efface le discours de Bossuet, c'est la création de l'Histoire ${ }^{15}$ ", tranchant la question du principe dualiste qui ouvrait l'Introduction à l'histoire universelle :

Avec le monde a commencé une guerre qui doit finir avec le monde, et pas avant ;

celle de l'homme contre la nature, de l'esprit contre la matière, de la liberté contre

la fatalité. L'histoire n'est pas autre chose que le récit de cette interminable lutte.

(IHU, p. 5)

16 Interminable lutte dont le paysage français, visage de la France, se fait le conservatoire ce pourquoi la formule du tour, du tour de France, qui gouverne tant Le Tableau de Michelet que les Voyages en France de Stendhal, était particulièrement appropriée en ce qu'elle permet d'enregistrer les éléments d'une dialectique que l'approche géographique qui sous-tend la progression, subsumant les contradictions, permet de résoudre parce que, par nature, elle est dépositaire de l'histoire tout entière, qui vient donner son sens à la nature.

\section{Le génie des lieux à l'horizon de l'histoire}

Si la nature a partie liée avec la fatalité, il s'ensuit que "l'intérêt du paysage ne suffit pas» mais qu'«il faut un intérêt moral ou historique» (VF, p. 468). Si Philippe L*** passe « deux heures délicieuses dans les chemins et sentiers le long de la Saône », c'est qu'il est « absorbé dans la contemplation des temps héroïques où Mme Roland a vécu », ces temps où «nous étions alors aussi grands que les premiers Romains» (MT, p. 74). Rien d'étonnant, dès lors, à ce que «la vue d'un fort beau paysage ", à Clermont, jette le touriste dans "l'épuisement moral»: c'est qu'alors la nature oblitère la civilisation, aussi notre voyageur d'en appeler à «la colonne antique la plus insignifiante » (MT, p. 170).

18 C'est que la référence romaine embraie immédiatement dans ces voyages sur une filiation républicaine et sur un corpus de valeurs qui tournent autour du bien public. "Arts de l'histoire ", les vestiges sont aussi les gardiens d'une morale incarnée. Ainsi de la Maison Carrée ou du Pont du Gard, tous lieux véritablement épiphaniques, quand «Les Romains faisaient de ces choses étonnantes, non pour inspirer l'admiration mais simplement et quand elles étaient utiles » $(M T, 365)$. Entendons à tous.

Mais Philippe $\mathrm{L}^{* * *}$ est loin de n'être que l'homme du passé et son rapport aux monuments historiques dit bien quels enjeux nouveaux ils recèlent: chez le touriste stendhalien, jamais un monument ne vient fossiliser l'environnement dans lequel il s'inscrit : ses leçons diffusent toujours, et c'est au présent qu'il parle. Sinon pour la réalité $d u$ présent, du moins pour son idéalité. Il acquiert ainsi une valeur d'exemplarité qui lui vient de la typologie dans laquelle il s'inscrit - pour le Pont-duGard, l'utilité publique, à laquelle est toujours associé le monde romain, porteur d'une idée de la citoyenneté d'autant plus forte qu'elle a été réactivée par la Révolution française, d'un « peuple-roi » (MT, p. 365) à l'autre.

20 Et pour Michelet aussi, dans ce Languedoc palimpseste de civilisations, la part des Romains est décisive, qui « ont enfoncé la plus profonde trace ; leur maison carrée, leur triple pont du Gard; leur énorme canal de Narbonne qui recevait les plus grands 
vaisseaux » (TF, p. 36). Sans compter que le droit romain compte explicitement comme une de ces « ruines » et est cause qu'ici « la présomption était toujours pour la liberté » (TF, p. 36).

21 Au tableau physique, fruit d'un savoir pluriel et actualisé, se surimpriment donc des représentations à fonction axiologique qui reposent sur une vision, et non pas sur la vue, à même d'assurer toutes les transfigurations. C'est le cas à Auch, ville mal bâtie que seul peut rédimer son passé romain, «Auguste » y ayant « laiss[é] une colonie qui se gouverna par ses propres lois et qui nomma ses magistrats. C'est bien plus de liberté que notre constitution actuelle en accorde à Auch » (VF, p. 682-683).

Mais que "l'histoire » puisse apparaitre comme « le triomphe progressif de la liberté » (IHU, p.5) ainsi que Michelet en posait le principe dans l'Introduction à l'histoire universelle suppose prescriptions et proscriptions qui dessinent, sous une "géographie différentielle du territoire ${ }^{16}$ ", un horizon historique orienté, et la liberté héritée des Romains avec leur culture de la res publica trouve son pendant négatif dans l'absolutisme qu'incarne Brest, élevée par l'autorité monarchique :

Brest, le grand port militaire, la pensée de Richelieu, la main de Louis XIV ; fort, arsenal et bagne, canons et vaisseaux, armées et millions, la force de la France entassée au bout de la France [...]. L'impression générale est grande, mais pénible [...]. J'y sens partout l'effort, et l'air du bagne et la chaîne du forçat. (TF, p. 7-8)

Palimpseste, le territoire cristallise le rapport aux mutations qui l'ont successivement affecté dans la longue durée de la construction nationale, que la Révolution est venue accomplir, achevant l'incorporation des parties dans le tout que forme désormais la nation en dépit d'évolutions locales dont Brest fait ici les frais alors que Paule Petitier relève plaisamment que Michelet "a réussi à faire l'apologie d'une centralisation apolitique, produit de la nation, du "génie de la France", et non de l'État ${ }^{17}$ ». De ce long processus d'assimilation, les indices sont multiples, qu'enregistrent tant Michelet que Stendhal - paysages, cultures et productions, physionomies et caractères, costumes et langues, couleurs et formes, coutumes et mœurs, politique et religion, fortunes et propriétés, architecture, littérature et folklore... - comme autant de témoins qui mesurent la marche vers une France, certes encore divisible, mais toujours plus une.

Pourtant, si l'approche mise en œuvre tient de l'enquête, l'observation ne tourne jamais au catalogue. Si constitution d'une banque de données il y a bien, les items entrent dans un système articulé que permet la comparaison (la formule "quelle différence avec ... » tient chez Stendhal du leitmotiv ${ }^{18}$ ). De là des morphèmes qui se dégagent du repérage des analogies et des différences et entrent dans un répertoire axiologique fonctionnel opératoire tant chez Michelet que chez Stendhal, responsable des découpages qui se donnent cours, en termes d'espace comme de civilisation.

Et tous deux reconnaissent un clivage fondamental : « Le tourbillon de la vie nationale a toute sa densité au Nord ; au Midi, les cercles qu'il décrit se relâchent et s'élargissent » (TF, p. 73), avance Michelet:

La vie forte est au Nord. Là s'est opéré le grand mouvement des nations. [...] Pour la France romaine et ibérienne, quelle que soit la splendeur de Marseille et de Bordeaux, elle ne regarde que le vieux monde de l'Afrique et de l'Italie. (TF, p. 3)

Et Stendhal de lui faire écho en revenant sur ces frontières :

Si vous voulez connaître la France moderne et civilisée, la France des machines à vapeur, placez votre tente au nord de la ligne de Besançon à Nantes; si c'est la France originale et spirituelle, la France de Montaigne que vous voulez voir, allez au midi de cette ligne. (MT, p. 93) 

p. 59), « les talents » et « les caractères qui assurent le succès dans le commerce » (VMF, p. 671-672). Au Sud, si « l'aspect charmant de la Méditerranée est d'accord avec le rôle qu'elle a joué dans le monde ", puisque «la Méditerranée a été le centre de la civilisation » (VF, p. 540), peut-on être et avoir été ? Michelet, on vient de le voir, opte pour la négative. De fait, si, chez Stendhal, le poids du Midi et sa représentativité doivent aussi être envisagés à l'aune du voyage qui lui est tout entier consacré, Michelet tranche, impitoyable: «Tout ce Midi si beau, c'est néanmoins, comparé au Nord, un champ de ruines. » (TF, p. 34.)

Pour autant, les lignes idéologiques, elles, semblent susceptibles de bouger. Car de quel lieu Philippe $\mathrm{L}^{* * *}$ parle-t-il en prononçant: «je pourrais remplir quatre pages de détails sur la prospérité de la France, et surtout des départements situés au nord de la ligne de Besançon à Nantes. Le Midi lui-même si encroûté, commence à se réveiller. » (MT, p. 153.) Qui parle par sa bouche, le touriste ou le marchand? Car touriste, le voyageur ne l'est pas tout à fait qui, ci-devant marchand de fer que ses affaires jette sur les routes, tient aussi du commis-voyageur. Dans ces années 1820-1840 qui sont celles de la révolution ferroviaire, le geste stendhalien, qui fait passer la partition au sein même de l'instance narrative organisatrice du point de vue, dit bien la volonté de tenir ensemble les deux France, celle du passé et celle de l'avenir, celle du Prince et celle du Marchand.

De fait, si le Midi est un champ de ruines, quand bien même, démon tentateur, il compte suffisamment de beaux restes pour dérouter vers son « génie naturel » (VMF, p. 671) un Michelet qui concède, sensible à « la poésie de ce destin du Midi », " Et moi aussi, j'y pleurerais comme Pétrarque au moment de quitter ces belles contrées » (TF, p. 44-45) au point de rendre son exposé un peu elliptique : «il me tarde de pousser au midi ; j’ai parlé des Celtes de Bretagne, je veux m'acheminer vers les Ibères, vers les Pyrénées. » (TF, p. 18 ;) la rationalité nordiste ${ }^{19}$ rebute continument un Stendhal que désole la " plate réalité toujours dégoûtante d'amour exclusif pour l'argent » (MT, p. 282) et le «Génie bien positif et réel » de la Flandre, «Lombardie prosaïque, à qui manquent la vigne et le soleil » (MT, p. 67), toujours marqueurs de civilisation, commande le retour $\mathrm{du}$ refoulé. Et il n'est jusqu'à la description du Bassin parisien, pourtant lieu géométrique de la dynamique centralisatrice micheletienne, qui ne soit informée d'accents que l'on pourrait croire stendhaliens :

Le bassin de la Seine et de la Loire est un pays remarquablement plat, pâle, indécis. Lorsque des pics sublimes des Alpes, des vallées sévères du Jura, des coteaux vineux de la Bourgogne, vous tombez dans les campagnes uniformes de la Champagne et de l'île de France au milieu de ces fleuves vagues et sales, de ces villes de craie et de bois, l'âme est saisie d'ennui et de dégoût. Vous voyez bien de grasses campagnes, de bonnes fermes et de bons bestiaux. Mais cette image prosaïque d'aisance et de bien-être ferait regretter la pauvre Suisse et jusqu'à la désolation de la campagne de Rome. (IHU, p. 50)

Si la France s'affirme comme le «lieu d'une synthèse dialectique entre les atouts quantitatifs (politiques, économiques et sociaux) du Nord et le prestige qualitatif (esthétique, éthique) du $\mathrm{Midi}^{20}{ }^{\prime}$, c'est encore chez notre touriste que ce processus de dépassement, marque d'une histoire vivante, est le plus concluant, notamment dans la transsubstantiation qui permet à Bordeaux d'être « et de bien loin aujourd'hui la plus belle ville de France » (VMF, p. 572), de réunir, en véritable Janus, sous des latitudes 
méridionales, la liberté de la Magna Carta et le libéralisme atlantique ${ }^{21}$, un passé d'humanités avec la modernité marchande ${ }^{22}$.

Une transfiguration réussie qui procède aussi de la position de celui à qui revient la performance du récit, fondamentalement informée par le romantisme et, comme telle, contestataire du palmarès qu'établiraient tant des classiques emperruqués que des bourgeois satisfaits, ouverte à une idée de la France à même d'admettre ensemble des réalités antinomiques. Celle de Juillet, en somme, qui, tout ensemble, donne un écrin au patrimoine culturel tout en lançant le pays sur les rails de l'industrialisation. Alors, « La société, la liberté, ont dompté la nature, l'histoire a effacé la géographie. Dans cette transformation merveilleuse, l'esprit a triomphé de la matière, le général du particulier, et l'idée du réel » (TF, p. 82).

\section{Du Nord au Midi : la mémoire culturelle scelle le patrimoine génétique de la France nouvelle}

Ce qui se joue à travers la représentation du territoire, d'un territoire sinon totalement un, puisqu'il est manifestement toujours divisible, mais en voie de centralisation, c'est la démonstration idéologique de la viabilité et de la supériorité du modèle unificateur jacobin, revu par 1830, qui en est le second volume et y met le point final au point que, dans la réédition de son texte, en 1869, Michelet en précise la genèse en rappelant qu'«il fut conçu d'un moment, l'éclair de Juillet. Dans ces jours mémorables, une grande lumière se fit, et j'aperçus la France ».

Si la France se laisse ainsi saisir, si son caractère organique apparaît alors, c'est, qu'enfin, cette fois, la Révolution est terminée, 1830 venant parachever 1789. Aussi le voyage d'études est-il résolument révolutionné quand c'est l'action fondatrice des héros de 1789 et de 1792 qui permet à Stendhal de constater qu'il est enfin possible de lever les blocages qui pesaient jusqu'alors sur un possible Voyage en France. Stendhal qui insiste clairement sur le rapport organique de cette France avec les acquis révolutionnaires, dont 1830 est pleinement solidaire :

On jouit enfin depuis 1830 des réformes introduites par Sieyès, Mirabeau, Carnot et les autres grands hommes de 1792. (MT, p. 152)

Force est d'enregistrer partout, au rythme des déplacements du marchand de fer, les preuves du dynamisme que connait le pays tout entier :

Je ne sais où trouver des termes prudents pour peindre la prospérité croissante dont la France jouit sous le règne de Louis-Philippe. J'ai peur de passer pour un écrivain payé.

À chaque pas, des maçons à l'œuvre, on bâtit une foule de maisons dans les villes, dans les bourgs, dans les villages; partout les rues se redressent. Dans les champs, on voit de tous les côtés creuser des fossés d'écoulement, bâtir des murs, planter des haies. (MT, p. 152).

La révision des positions sur le régime honni du "plus fripon des Kings » est sévère, quand le Barème Couronné fait la démonstration objective des changements et des progrès apportés, que les paysages enregistrent et dévoilent aux yeux de tous. La vertu pédagogique du voyage vaut alors révélation de l'état de la France. Si Paule Petitier qualifie le Tableau de la France d'« œuvre d'adhésion ${ }^{23}$ ", la leçon, nolens volens, paraît bien être la même pour celui que donne Stendhal. 
36 Alors peut s'opérer une appropriation de la patrie, quand la connaissance du pays sert l'unité de la nation et que l'engouement pour le patrimoine national marche d'un même pas. L'évidence de la singularité française accroît l'intérêt de mieux connaître la patrie pour en explorer la mémoire avec les fondements de l'héritage mémoriel.

Mais, pour cela, il s'impose de revenir à l'origine, et de s'incorporer d'abord les changements advenus. Victor Hugo ne fait pas autre chose en 1874 quand, remontant le cours des temps jusqu'en Quatrevingt-treize, il suit les métamorphoses du pays dans la naissance de la nation. Ce sont les armées bleues venues réduire la guerre civile et unifier la nation, et qui s'attirent cette réponse d'une Michelle Fléchard égarée :

- Quelle est ta patrie?

- Je ne sais pas, dit-elle.

- Comment, tu ne sais pas quel est ton pays?

$-\mathrm{Ah} ! \mathrm{mon}$ pays. Si fait.

- Eh bien, quel est ton pays?

La femme répondit :

- C'est la métairie de Siscoignard, dans la paroisse d'Azé.

Ce fut le tour du sergent d'être stupéfait. Il demeura un moment pensif, puis il

reprit :

- Tu dis?

- Siscoignard.

- Ce n'est pas une patrie, ça.

- C'est mon pays.

Et la femme, après un instant de réflexion, ajouta :

- Je comprends, monsieur. Vous êtes de France, moi je suis de Bretagne.

- Eh bien?

- Ce n'est pas le même pays.

- Mais c'est la même patrie! cria le sergent ${ }^{24}$.

Avant que Victor Hugo n'en énonce la portée universelle :

Pays, Patrie, ces deux mots résument toute la guerre de Vendée; querelle de l'idée locale contre l'idée universelle; paysans contre patriotes ${ }^{25}$.

On saisit là la diversité des échelles qui mesurent tant la construction du territoire national que le vécu des habitants, dont certains demeurent inscrits dans les limites de leur espace vécu, du terroir légué par leurs ancêtres. De ce point de vue, le cas de cet Ouest armoricain apparaît, comme nul autre, porteur de l'écart maximal, quand le conservatoire paysager vaut prise de position politique. Michelet pousse loin cette lecture, qui relève, dans son Tableau de la France, tous les indices - physiques et imaginaires - qui rattachent ces pays à un espace obscurantiste et passéiste :

Je n'oublierai jamais le jour où je partis de grand matin pour Auray, la ville sainte des chouans, pour visiter à quelques lieues la ville druidique de Loc Maria Ker et de Carnac. Le premier de ces villages, à l'embouchure de la sale et fétide rivière d'Auray, avec ses îles du Morbilhan, plus nombreuses qu'il n'y a de jours dans l'an, regarde par-dessus une petite baie la plage de Quiberon, de sinistre mémoire. (TF, p. $12^{26}$ )

La nature elle-même, dans sa noirceur et sa laideur, ses chaos de pierres informes, sa pestilence même, affiche ici le choix de la contre-révolution, qui a échoué à Quiberon en 1795. Autant de traits qui étrangent la Bretagne, «triste et sauvage» (TF, p. 4-5) pour Michelet, triste et grise pour Stendhal (MT, p. $244 ;$ p. 306), à l'unité supérieure de la patrie, rationnelle et progressiste, si chère à Michelet :

La personnalité, l'unité, c'est par là que l'être se place haut dans l'échelle des êtres. [...] La solidarité [des] parties [...], c'est là la supériorité sociale. C'est celle de la France, le pays du monde où la nationalité, où la personnalité nationale se 
rapproche le plus de la personnalité individuelle.

Diminuer, sans la détruire, la vie locale, particulière, au profit de la vie générale et commune, c'est le problème de la sociabilité humaine [...]. Le peuple le mieux centralisé est aussi celui qui par son exemple, et par l'énergie de son action, a le plus avancé la centralisation du monde.

Cette unification de la France, cet anéantissement de l'esprit provincial est considéré fréquemment comme le simple résultat de la conquête des provinces. La conquête peut attacher ensemble, enchaîner des parties hostiles, mais jamais les unir. [...] ces provinces, diverses, [...] se sont [...] senties solidaires [...].

Ainsi s'est formé l'esprit général, universel [...]. L'esprit local a disparu [...]; l'influence du sol, du climat, de la race, a cédé à l'action sociale et politique. La fatalité des lieux a été vaincue [...]. La société, la liberté, ont dompté la nature, l'histoire a effacé la géographie. Dans cette transformation merveilleuse, l'esprit a triomphé de la matière, le général du particulier et l'idée du réel. [...] la société humaine [...] tend à s'affranchir sans cesse des misères de l'existence locale, à atteindre la haute et abstraite unité de la patrie. (TF, p. 82-86)

41 La leçon du Tableau de la France voit ainsi le génie français s'incarner dans l'unité que réalisent, ensemble, la géographie et l'histoire, l'une subsumant l'autre puisque "l'histoire est d'abord toute géographie » $(T F, 82)$ au seuil de l'analyse avant que la péroraison ne porte que "l'histoire a effacé la géographie » $(T F, 160)$, la géographie comme l'histoire étant d'ailleurs productrices de cette centralisation harmonieuse que la Révolution a relancée sur des bases rationnelles et nationales. Une fusion qui dit aussi la logique épistémique toute historique qui guide le $\mathrm{XIX}^{\mathrm{e}}$ siècle et qui préside à ses élaborations conceptuelles.

Et même si le type de développement observé n'est pas celui que les libéraux avaient pu espérer, le constat est là de l'équilibre incontestable auquel le pays est parvenu. Le possible voyage en France signe alors l'aboutissement du processus de réconciliation entamé par l'Empire. Et ce n'est pas un hasard s'il devient vraiment praticable avec la monarchie de Juillet, quand essor économique et développement mémoriel servent ensemble à l'exaltation de la France nouvelle issue du chantier révolutionnaire. Philippe $\mathrm{L}^{* * *}$, marchand de fer et touriste, signale et signe ce grand tournant que sacralise solennellement le retour des Cendres en 1840.

C'est l'appropriation de ce passé recomposé, de la mémoire collective inscrite au cœur même du pays, dont le voyage en France peut désormais assurer la promotion, ouvrant les voies à la vulgate que l'on voit triompher dans l'enseignement de la seconde moitié $\mathrm{du} \mathrm{XIX}^{\mathrm{e}}$ siècle et que les manuels scolaires vont diffuser à profusion, dans le prolongement de la loi Guizot sur l'Instruction primaire du 28 juin 1833 dont l'article premier prévoit que «L'instruction primaire supérieure comprend nécessairement [...] les éléments de l'histoire et de la géographie, et surtout de l'histoire et de la géographie de la France ». La route était pavée pour que, avant même que Flaubert ne l'annonce à Louise Colet, « Yvetot va[ille] Constantinople ${ }^{27}$ ». 


\section{NOTES}

1. Toutes les références à ces textes, abrégés en $M T, V F$ et $V M F$, renvoient à l'édition de V. Del Litto, Stendhal, Voyages en France: Mémoires d'un touriste, Voyage en France, Voyage dans le Midi de la France, Paris, Gallimard, coll. « Bibliothèque de la Pléiade », 1992.

2. D. Poulot, Musée, nation, patrimoine. 1789-1815. Paris, Gallimard, coll. «Bibliothèque des Histoires », 1997, p. 13.

3. Le 21 octobre 1830, un rapport de Guizot conclut à la création d'un poste d'inspecteur général des Monuments historiques. Il sera suivi, en 1834, par l'institution d'un Comité historique des arts et des monuments rebaptisé, en 1837, Commission des monuments historiques, qui recense les bâtiments méritant protection.

4. Le titre complet de ce volume est Tableau de la France : condensé d'histoire de France des origines à 1830. Nos références à cet ouvrage renverront à l'édition Tableau de la France. Géographie physique, politique et morale par Jules Michelet, Paris, A. Lacroix et $C^{\text {ie }}, 1875$ [abrégé en TF]. Et, pour L'Introduction à l'Histoire universelle, Paris, Hachette, 1831 [abrégé en IHU].

5. Voir P. Petitier, La Géographie de Michelet. Territoire et modèles naturels dans les premières œuvres de Michelet, Paris, L'Harmattan, 1997, p. 153-166: «le territoire c'est [...] l'histoire visible, fixée, déployée, le livre déjà écrit de l'historien », p. 158.

6. J. Michelet, Histoire du XIX siècle, dans Euvres complètes, t. XXI (1872-1874), Paris, Flammarion, 1982, p. 57.

7. P. Petitier, ouvr. cité, p. 192. Pour la citation de Michelet, Bibliothèque historique de la ville de Paris, Fonds Michelet, Ms A 3890, fo 13.

8. A. Monglond, La France révolutionnaire et impériale. Annales de bibliographie méthodique et description des livres illustrés, Grenoble, Arthaud, 1930.

9. MT, p. 94-97 et 298-301.

10. Hors, toutefois, d'une stricte orthodoxie puisque, dans son Journal de 1831 où il consigne les réflexions que le terrain lui inspire, Michelet relève que « le terroir fait plus que la race » et tout indique qu'il est alors sur le point de dépasser cette théorie.

11. Je renvoie ici à l'article capital de G. Rannaud, "Le naturel et ses équivoques ", dans Ph. Berthier (éd.), Stendhal. Colloque de Cerisy-la-Salle (30 juin-16 juillet 1982), Aux amateurs de livres, 1984, p. 103-118.

12. «Je n'ai rien rencontré en France dans ce voyage qui ait autant de style, c'est-à-dire qui parle tant à l'âme, que ce petit palais » $(V M F, 663)$. C'est poser là l'épineux problème de la frontière : de même que Michelet fait silence sur l'Alsace, au motif qu'il la rattache au monde germanique alors, qu'au contraire, il s'étend sur la Belgique, annexée à la culture française, de même Stendhal nationalise le pays basque espagnol.

13. J. Michelet, Introduction à l'histoire de France dans IHU, p. 49-50.

14. "Quel dommage", s'écrie Philippe $L^{* * *}$ sur la route de Langres à Dijon, "qu'une fée bienfaisante ne transporte pas [au mont Valérien] quelqu'une de ces terribles montagnes des environs de Grenoble!» (MT, 59.) En 1868, dans La Montagne, Michelet lui donnera raison en portant condamnation de Paris pour n'avoir pas de montagne. Désabusé par l'évolution politique, Michelet revient alors du bassin Parisien, lieu géométrique de la centralisation française et en vient, contre un jacobinisme fourvoyé, à célébrer la Montagne, faisant du massif du Saint-Gothard le cœur d'une Europe en devenir.

15. J. Michelet, Nos fils, Paris, Librairie internationale, Lacroix, Verboeckhoven et $C^{\text {ie }}, 1870$, p. 194.

16. Au sens où l'entend R. Chartrier dans "La ligne Saint-Malo-Genève ", dans Les Lieux de mémoire, II, P. Nora (éd.), Paris, Gallimard, coll. « Quarto », 1997, p. 2817.

17. P. Petitier, ouvr. cité, p. 59. 
18. Par exemple $M T$, p. 207, p. 306, p. 310, p. 358 ; VF, p. 535, p. 456.

19. À l'inverse, le Midi n'est pas une terre de rationalisme économique et Michelet de rappeler l'indignation qui saisit Arthur Young lors de son voyage dans le Midi en Auvergne, où officie encore souvent «la petite charrue du Midi qui égratigne à peine le sol », la Loire opérant la limite, $T F$, p. 24 et note 5.

20. Y. Le Scanff, "L'origine littéraire d'un concept géographique : l'image de la France duelle ", Revue d'histoire des Sciences humaines, 2001/2, n 51, p. 61-93.

21. Je ne reprends pas ici les étapes d'une démonstration que l'on retrouvera dans L. Lévêque, "Géo-graphie ou ego-graphie: au détour du paysage-miroir dans les Mémoires d'un touriste, le Voyage en France et le Voyage dans le Midi de la France ", L'Année Stendhalienne, 3, 2004, p. 84.

22. Si chez Michelet, Bordeaux, «longtemps capitale de la France anglaise » et «tournée par l'intérêt de son commerce vers l'Angleterre, vers l'océan, vers l'Amérique » est tout entier anglisé, « La Garonne » étant même « deux fois plus large que la Tamise à Londres » (TF, p. 27), le Poitou, «bataille du Midi et du Nord» (TF, p. 18), peut un temps prétendre tenir la balance entre Nord et Sud. "Terre de contraste, "pays du mélange ", "mêlé de droit romain et de droit coutumier », il donne "ses légistes au Nord, ses troubadours au Midi». Comme sa mélusine, "assemblage de natures diverses", il est «moitié homme, moitié serpent» (TF, p. 18). Mais, " génie mixte et contradictoire» (TF, p. 19), le Poitou a beaucoup entrepris mais rien achevé. Il lui manque une idée directrice dont l'île de France est seule à faire montre.

23. P. Petitier, ouvr. cité, p. 210. Elle le lie aux fortunes de carrière qu'il connaît alors: nomination à la tête de la section historique des Archives, maître d'histoire de la princesse Clémentine, nomination au Collège de France et à l'Académie...

24. V. Hugo, Quatrevingt-treize, dans E Euvres complètes, Romans, III, Paris, Robert Laffont, coll. « Bouquins », 1985, p. 792-793.

25. Ibid., p. 925.

26. Voir P. Petitier, ouvr. cité, p. 206.

27. « Yvetot, donc, vaut Constantinople », Lettre du 25 juin 1853, dans G. Flaubert, Correspondance, II, Paris, Gallimard, coll. «Bibliothèque de la Pléiade », 1980, p. 362. Voir également N. Mozet, "Yvetot vaut Constantinople. Littérature et géographie en France au XIX ${ }^{\mathrm{e}}$ siècle ", Romantisme, $\mathrm{n}^{\circ}$ 35, 1982, p. 91-114.

\section{RÉSUMÉS}

Cet article entend défendre l'idée que les différents Voyages en France - Mémoires d'un touriste, Voyage en France et Voyage dans le Midi de la France - que Stendhal rédige en 1838 sont étroitement tributaires d'un moment historique connu comme le "Moment Guizot » qui, au tournant des années 1830 , entame une politique de réconciliation nationale laquelle rencontre l'agenda d'un Henri Beyle qui engage sa réincorporation dans une patrie quittée huit ans auparavant. Si les tensions sont vives pour l'auteur de Lucien Leuwen qui flétrissait le régime du " plus fripon des kings ", elles témoignent d'une réalité dont prenait acte alors tant Charles Dupin dans ses Forces productives de la France que Michelet dans son Tableau de la France de 1833, plaidant pour un spectaculaire redressement auquel Stendhal, nolens volens, apporte sa caution.

In this article, the hypothesis that the various Travels Through France - Memoirs of a Tourist, Travels Through France and Travels Through the South of France - written by Stendhal in 1838 are closely 
related to a historical moment known as the "Guizot Moment" is raised. François Guizot, one of the most prominent French politicians of the time - if not the most -, initiated, at the turn of the 1840 s, a policy of national reconciliation that coincided with the wishes and thoughts of Henri Beyle, as the latter was getting re-acquainted with the habits and customs of a country he had left eight years before. Acute tensions then pervaded the works of the author of Lucien Leuwen - who had condemned the régime of "the most knavish of kings"-, but they give evidence of a reality then acknowledged by Charles Dupin and Jules Michelet alike -in their respective works, The Productive Forces in France and A Picture of France (1833). Both were in favour of a spectacular upturn to which Stendhal, willingly or unwillingly, gives his backing.

\section{AUTEUR}

\section{LAURE LÉVÊQUE}

Université de Toulon

BABEL, EA 2649

Laure Lévêque est professeur de littérature française à l'université de Toulon et membre du laboratoire Babel (EA 2649). Elle travaille sur l'écriture de l'histoire dans le long XIX siècle et s'intéresse notamment à la part des élaborations imaginaires et idéologiques dans la transmission et la construction des référents culturels et à la sélection des composantes appelées à former le fonds d'une culture commune, qu'elle aborde dans une perspective résolument transdisciplinaire. Sur ces questions, elle est l'auteur de Le Roman de l'histoire. 1780-1850 (L'Harmattan, 2001) et de Penser la nation. Mémoire et imaginaire en révolutions (L'Harmattan, 2011). 